\title{
Influence of rice varieties on sensory profile and consumer acceptance for frozen-cooked rice
}

\author{
Han Sub Kwak ${ }^{1,2}$, Yoonhwa Jeong, ${ }^{1,2}$, Misook Kim ${ }^{1,2 *}$ \\ ${ }^{1}$ Department of Food Science and Nutrition, Dankook University, Yongin-si, 448-701 Korea; ${ }^{2}$ Institute of Global Food Industry, Dankook \\ University, Yongin-si, 448-701 Korea
}

\section{A B S T R A C T}

\begin{abstract}
The objectives of this study were to generate sensory profiles by descriptive analysis, to access consumer acceptability by consumer acceptance test, and to determine the driving sensory attributes for frozen-cooked rice made by five difference rice varieties. Rice varieties resulted in significant differences in the sensory profiles of the frozen-cooked rice samples. Sixteen descriptive attributes - aroma (rice bran, raw rice, tap water), taste (sweet, bitter), flavor (rice cake, roasted), texture (glutinousness, firmness, cohesiveness of mass, particle feeling, moistness), and appearance (amount of embryo buds, glossiness, rice shape, whiteness, size of rice) were used as descriptive terms for the samples. Oryza sativa L. var. Ilpum was the most accepted variety when processed to frozen cooked rice and should therefore be the first option for processing. Partial least square regression analysis found no drivers of liking and five drivers of disliking for frozen-cooked rice: Rice bran aroma, glutinousness, firmness, particle feeling and amount of embryo buds.
\end{abstract}

Key words: Consumer test; Descriptive analysis; Frozen-cooked; Rice; Variety

\section{INTRODUCTION}

Medium and short grain rice (Oryza sativa L.) is the staple food of northeastern Asian countries including Korea, Japan and northern China. Recently, the Unites State has become one of the major countries growing medium and short grain rice. These rice varieties are called 'sticky rice' because the rice kernels are attached together and have a glutinous texture after cooking. These rice varieties are mainly consumed after cooking or steaming in most countries. However, it is difficult to maintain cooked rice quality after cooking because of staling (Yu et al., 2010). Freezing techniques have been applied to bread products (Barcenas and Rosell, 2006), cooked starchy convenience foods (De Kock et al., 1995) and pasta (Olivera and Salvadori, 2009) to maintain food quality after cooking in western countries. Applying a freezing technique would be one option to extend storage periods with consistent quality for cooked rice, and change the notion of home-cooking of rice to the purchase cooked rice at the grocery market as ready-to-eat products.

Frozen-cooked rice processing is relatively simple in comparison with aseptic-packaged cooked rice processing
(Ji, 2010). The facility for aseptic-packaged cooked rice is expensive (approximately 20 million dollars in Korea) and requires an aseptic-packaging processing, which required extensive to prevent microorganism contamination. In addition, processing manipulation for the production of different products is difficult. On the other hand, a frozen-cooked rice processing facility is a relatively cheap (approximately 5 million dollars in Korea) and small processing line. For safety, microorganism contamination can be easily prevented by freezing after cooking. Ease of processing control enables producers to manufacture various frozen-cooked rice items using only one processing line. The frozen cooked rice market in Korea is increasing by $20-30 \%$ of revenue every year by increasing from 2.3 million dollars in 2010 to 12 million dollars in 2013 (Food News, 2014; Ji, 2010). Potential growth is eventually expected to reach 200 million dollars, considering the 500 million dollars Japanese market for frozen-cooked rice in 2005 (Park, 2008).

Although the growing popularity of frozen-cooked rice as well as the consistent growth of aseptic-packaged cooked rice have been noted (Kum, 2010), there are

\footnotetext{
${ }^{*}$ Corresponding author:

Misook Kim, Department of Food Science and Nutrition, Dankook University, 152 Jukjeon-ro, Natural Science Bldg \#218, Yongin-si, 448-701 Korea. E-mail: mkim5@dankook.ac.kr, Tel: 82-31-8005-3986, Fax: 82-31-8021-7200

Received: 26 April 2015;

Revised: 18 October 2015;

Accepted: 20 October 2015;

Published Online: 20 October 2015
} 
few studies involving sensory evaluations of processed cooked rice products. Most of the published studies used steamed rice for their evaluations (Suwansri et al., 2002; Yau and Huang, 1995; Meullenet et al., 2001). Quality and sensory characteristics of a rice variety (Juanbyeo) developed for aseptic-packaged cooked rice were reported in Korea (Oh et al., 2010). Three different processing methods for cooked rice - aseptic-packaged, steamed and frozen - were compared in terms of their sensory profiles and consumer acceptance, and showed similar consumer acceptability between home-made and frozen cooked rice (Kwak et al., 2013a). Kwak et al. (2015b) recently reported that the drivers of liking for aseptic-packaged cooked rice were rice cake flavor, sweetness, roasted aroma and flavor, cohesiveness of mass, deformation and shininess. However, to the authors' knowledge, there have been no studies on the sensory profiles and consumer acceptability of frozen-cooked rice to date. It is necessary to identify sensory profiles, measure consumer acceptance, and to determine driving sensory attributes in order for the industry to produce high quality frozen-cooked rice as frozen-cooked rice market expanding.

\section{MATERIALS AND METHODS}

\section{Rice variety}

Five rice varieties grown in a commercial rice paddy in Korea were harvested in 2012. Oryza sativa L. var. Boramchan (BO) had strong disease endurance and higher yield in comparison with regular varieties (KiYong et al., 2012). Oryza sativa L. var. Chucheong $(\mathrm{CH})$ was one of the major rice varieties in Korea and the most commonly consumed variety at home due to its high quality (Oh et al., 2010). Oryza sativa L. var. Sindongin (SI) was a rice variety widely grown in the southwestern part of Korea, an area known as the granary of Korea. Oryza sativa L. var. Ilpum (IL) had small, round shaped rice kernels. The advantages of these varieties was that the consumer acceptance of frozen-cooked SI and IL rice were similar to home-cooked rice (Kwak et al., 2013a). Oryza sativa L. var. Ilmi (IM) was the variety typically used for frozen-cooked rice production, with $8 \%$ of total production in Korea. The rice varieties was purchased from the local rice processing complexes as brown rice and stored in a $4{ }^{\circ} \mathrm{C}$ low-temperature warehouse. The brown rice kernels were polished to white rice kernels ( $92 \%$ weight of brown rice) using a rice polishing machine (Model MC-250, Wakayama Co. Ltd., Wakayama, Japan).

\section{Quality characteristics of polished rice kernels}

The ratio of head rice after milling was determined using a lab size rice inspector (RN-200, Kett Electronic Laboratory, Tokyo, Japan). Approximately 1000 kernels were placed in the inspector and the ratio of head rice was calculated.
The moisture content of the rice kernels was measured by the AOAC method (AOAC, 2002). Rice (10.0 g) was dried overnight in a dry oven $\left(105^{\circ} \mathrm{C}\right)$ and the weight was measured. The difference in weight before and after drying was divided by the initial weight to calculate the percentage of moisture content. Rice freshness was measured using a rice freshness analyzer (Shinsensa, Satake Corporation, Hiroshima, Japan) in order to compare the activity of peroxidase as an indicator of the degree of freshness (Chen and Chen, 2003). Measurements were triplicated and averaged.

\section{Frozen-cooked rice preparation}

Frozen-cooked rice was prepared by Kwak's method (Kwak et al., 2013a). Briefly, polished rice (1000.0 g) was washed three times using tap water and then soaked in tap water for $80 \mathrm{~min}$. After draining, the soaked rice and filtered spring water (Sparkle, Sparkle, Co. Ltd., Seoul, Korea) were placed in an electronic rice cooker (CR-1502-G, CuCu Homesys Co., Yangsan, Korea). Rice was cooked using a regular cooking option and left for an additional $15 \mathrm{~min}$ for carryover cooking. Cooked rice samples above $1 \mathrm{~cm}$ from the bottom and below $1 \mathrm{~cm}$ from the top were mixed together. Two hundred grams of cooked rice were transferred to a plastic package and wrapped twice with a plastic wrap. The packaged cooked rice samples were quick-frozen for one hour using a rapid-freezer at $-70{ }^{\circ} \mathrm{C}$ (Blast chiller, IRINOX, Treviso, Italy) and the samples were stored at $-20^{\circ} \mathrm{C}$ for two weeks prior to the descriptive analysis and consumer acceptance test.

\section{Descriptive analysis}

Frozen-cooked rice samples were heated for three minutes using a microwave (RE-C24RWS, Samsung Electronics Co., Ltd., Seoul, Korea) at 700W. Approximately $66 \mathrm{~g}$ of frozen-cooked rice was transferred to a $150 \mathrm{~mL}$ plastic cup and covered with a lid. The samples were placed in pottery cups and the cups were placed in thermos lunch boxes (AL-500, Woonam Industry, Seoul, Korea), which were kept in a warmer $\left(70{ }^{\circ} \mathrm{C}\right)$, to minimize temperature change. All samples were kept in a warmer $\left(70{ }^{\circ} \mathrm{C}\right)$ prior to the descriptive analysis. Panelists used chopsticks for sample tastings. Three-digit random numbers were displayed on the thermos lunch box for sample identification.

Descriptive analysis was conducted with 8 trained panelists (females, ages $34-51$, living in the Seoul capital area, Korea). The panelists had at least 360 hours of experience in Quantitative Descriptive Analysis (QDA) within the past three years. Trainings and evaluations for frozen-cooked rice were carried out for 12 hours. Training was conducted for 10 hours and two final evaluations were performed for two hours. The panelists were asked to swallow the samples after tasting for a unified tasting procedure. Filtered spring water 
(Sparkle, Sparkle Co. Ltd., Seoul, Korea) was used as a palate cleanser. In the first session, the panelists tasted 5 samples and generated descriptive attributes for aroma, taste, flavor, texture and appearance. Definitions and references for the attributes were also discussed. During the next 4 sessions, panelists evaluated references and discussed the attributes to consolidate them to a number below 20 . Finally, 17 attributes were determined (Table 1). The attributes and intensity ratings for each attribute were determined over the training of three sessions. The panelists rated the attribute intensities using a 16-point category scale, which was labeled as ' $0=$ not at all and $15=$ extremely strong'. The panelists compared their intensity ratings and discussed attributes that had different rating patterns. Finally, panelists participated in a practice evaluation to familiarize them with the final testing procedure. The evaluation was conducted in individual booths with a computerized sensory data collection system (Sens Mine, Sensometrics, Seoul, Korea) under a red lighting system. The appearance attributes were evaluated in a light box (Superlight, Boteck, Siheung, Korea) with 'day light' setup. The results were discussed in the next session to minimize variations among panelists. Re-training was performed for attributes that showed inconsistent rating patterns among the panelists during the practice evaluation. Final evaluations

Table 1: Attribute, definition, reference and evaluation process of 17 attributes for frozen cooked rice samples from 5 rice cultivars

\begin{tabular}{|c|c|c|c|}
\hline Attribute & Definition & Reference & Evaluation process \\
\hline \multicolumn{4}{|l|}{ Aroma } \\
\hline Rice bran & $\begin{array}{l}\text { Aroma perceived from } \\
\text { rice bran }\end{array}$ & Rice bran & $\begin{array}{l}\text { Measure the highest intensity of rice bran aroma when smelling } \\
\text { samples }(0=\text { no perception, } 15=\text { extremely strong) }\end{array}$ \\
\hline Raw rice & $\begin{array}{l}\text { Aroma perceived from } \\
\text { raw rice }\end{array}$ & Polished rice & $\begin{array}{l}\text { Measure the highest intensity of raw rice aroma when smelling } \\
\text { samples }(0=\text { no perception, } 15=\text { extremely strong) }\end{array}$ \\
\hline Tap water & $\begin{array}{l}\text { Aroma perceived from tap } \\
\text { water before boiling }\end{array}$ & $\begin{array}{l}\text { Tap water } \\
\text { (just after boiling) }\end{array}$ & $\begin{array}{l}\text { Measure the highest intensity of tap water aroma when smelling } \\
\text { samples ( } 0=\text { no perception, } 15=\text { extremely strong) }\end{array}$ \\
\hline \multicolumn{4}{|l|}{ Taste } \\
\hline Sweet & Sweet taste & $\begin{array}{l}2 \%\left(w^{-1}\right) \text { sugar } \\
\text { solution }\end{array}$ & $\begin{array}{l}\text { Measure intensity of sweetness after } 5-8 \text { times of chewing } \\
(0=\text { no perception, } 15=\text { extremely strong })\end{array}$ \\
\hline Bitter & Bitter taste & $\begin{array}{l}0.03 \%\left(w v^{-1}\right) \\
\text { caffeine solution }\end{array}$ & $\begin{array}{l}\text { Measure intensity of bitterness after } 5-8 \text { times of chewing } \\
(0=\text { no perception, } 15=\text { extremely strong) }\end{array}$ \\
\hline \multicolumn{4}{|l|}{ Flavor } \\
\hline Rice cake & Flavor of rice cake & $\begin{array}{l}\text { Sliced steamed } \\
\text { rice cake }\end{array}$ & $\begin{array}{l}\text { Measure intensity of steamed rice cake flavor after } 3-5 \text { times of } \\
\text { chewing }(0=\text { no perception, } 15=\text { extremely strong) }\end{array}$ \\
\hline Roasted & $\begin{array}{l}\text { Flavor of dried and roasted } \\
\text { cooked rice }\end{array}$ & Puffed rice snack & $\begin{array}{l}\text { Measure intensity of roasted flavor after } 3-5 \text { times of } \\
\text { chewing ( } 0=\text { no perception, } 15=\text { extremely strong) }\end{array}$ \\
\hline \multicolumn{4}{|l|}{ Texture } \\
\hline Glutinousness & Stickiness of cooked rice & Caramel & $\begin{array}{l}\text { Measure intensity of glutinousness when chewing using molars } \\
\text { after } 3-5 \text { times of chewing } \\
\text { ( } 0=\text { not at all, } 15=\text { extremely glutinousness) }\end{array}$ \\
\hline Firmness & $\begin{array}{l}\text { Force to cut cooked rice } \\
\text { kernels using the front teeth }\end{array}$ & Roasted peanut & $\begin{array}{l}\text { Measure the force when cutting rice kernel using front teeth } \\
\text { ( } 0=\text { extremely softness, } 15=\text { extremely firmness) }\end{array}$ \\
\hline $\begin{array}{l}\text { Cohesiveness } \\
\text { of mass }\end{array}$ & $\begin{array}{l}\text { Formation of mass when } \\
\text { chewing the sample }\end{array}$ & Steamed rice cake & $\begin{array}{l}\text { Chew } 2-3 \text { times of cooked rice using molars and evaluate }(0=\text { not } \\
\text { at all, } 15=\text { extremely) }\end{array}$ \\
\hline Particle feeling & $\begin{array}{l}\text { Amount of left-over particles } \\
\text { after shallow cooked rice }\end{array}$ & Puffed rice kernel & $\begin{array}{l}\text { Feeling of left-over rice particles after swallowing puffed rice kernel } \\
(0=\text { not at all, } 15=\text { extremely })\end{array}$ \\
\hline Moistness & $\begin{array}{l}\text { Perception of moisture } \\
\text { content when pressing tofu } \\
\text { in the mouth }\end{array}$ & Tofu & $\begin{array}{l}\text { Perceive moistness when pressing cooked rice kernels } \\
(0=\text { extremely low, } 15=\text { extremely high) }\end{array}$ \\
\hline \multicolumn{4}{|l|}{ Appearance } \\
\hline $\begin{array}{l}\text { Amount of } \\
\text { embryo buds }\end{array}$ & $\begin{array}{l}\text { Amount of embryo bud on } \\
\text { the rice }\end{array}$ & $\begin{array}{l}\text { Commercial } \\
\text { aseptic-packaged } \\
\text { cooked rice }\end{array}$ & $\begin{array}{l}\text { Measure how many embryo buds were observed in comparison } \\
\text { with the surface of commercial aseptic-packaged cooked rice }\end{array}$ \\
\hline Glossiness & $\begin{array}{l}\text { Glossiness of cooked rice } \\
\text { kernel }\end{array}$ & $\begin{array}{l}\text { Surface of commercial } \\
\text { aseptic-packaged rice } \\
\text { porridge }\end{array}$ & $\begin{array}{l}\text { Measure the intensity of glossiness of cooked rice surface under } \\
\text { lightening box } \\
\text { (1=no glossiness, } 15=\text { extremely glossiness) }\end{array}$ \\
\hline Rice shape & $\begin{array}{l}\text { Degree of whole shape of } \\
\text { rice kernels }\end{array}$ & $\begin{array}{l}\text { Surface of commercial } \\
\text { aseptic-packaged rice } \\
\text { porridge }\end{array}$ & $\begin{array}{l}\text { Measure the degree of whole shape of rice kernels } \\
(0=\text { no whole shape kernel, } 15=\text { perfect whole shape rice kernel) }\end{array}$ \\
\hline Whiteness & Degree of white color & $\begin{array}{l}\text { Standard white color } \\
\text { (Hex triplet: \#FFFFFF) }\end{array}$ & $\begin{array}{l}\text { Measure the intensity of standard white color on the computer } \\
\text { screen }\end{array}$ \\
\hline Size of rice & Size of intact rice & $\begin{array}{l}\text { Commercial } \\
\text { aseptic-packaged } \\
\text { cooked rice kernel }\end{array}$ & $\begin{array}{l}\text { Measure the size of a rice kernel under lightening box } \\
\text { (1=extremely small, } 8=\text { sample as commercial, } 15=\text { extremely large) }\end{array}$ \\
\hline
\end{tabular}


were carried out twice using the same set-up as the practice evaluation. Samples were presented according to the mutually orthogonal Latin square (MOLS) design to minimize carryover effect (Wakeling and MacFie, 1995). Side-by-side scale presentation method was used to allow the panelists to compare the intensities of each attribute within the samples (Mazzuchelli and Guinard, 1999).

\section{Consumer acceptance test}

The consumer acceptance test was conducted with 52 subjects (females, ages 29 - 49) living in the Seoul capital area in Korea. Consumers were instructed on the purpose of the test, how to evaluate the samples, testing procedure, and how to use a 15-point category scale (Kwak et al., 2013b; 2015b) for the frozen-cooked rice evaluation. The 15 -point category scale was labeled as ' $1=$ dislike extremely, $8=$ neither like nor dislike, and 15=like extremely'. Overall acceptance and acceptance of appearance, taste and texture were assessed. Samples were presented according to Williams' design for 5 samples to minimize carry-over effect (Williams, 1949). Samples were served one-by-one with 7-minute intervals to avoid sample comparisons during the test and to give panelists enough time for evaluation and recovery.

Each sample was heated with a microwave (C24RWS, Samsung Electronics Co., Ltd.) for three minutes. The sample was then placed in a white colored pottery bowl. One bowl was served to three consumers. Consumers transferred approximately $60 \mathrm{~g}$ of the sample to a $190 \mathrm{~mL}$ paper cup for tasting. Consumers used chopsticks and were asked to swallow the sample after the evaluation. Filtered spring water (Sparkle, Sparkle Co., Ltd, Seoul, Korea) was served as a palate cleanser. The consumer acceptance test was carried out in a consumer testing room of the recruiting company in one session. Fluorescent lighting was used and the temperature of the testing room was maintained at $24^{\circ} \mathrm{C}$. Conversation and cell phone use were strictly prohibited during the test. Monetary compensation was provided after the test.

\section{Statistical analyses}

XLSTAT (version 2012, Addinsoft, Paris, France) was used for all statistical analyses. One-way analysis of variance (ANOVA) was performed for the quality characteristics of rice kernels. Seventeen descriptive attributes were analyzed by 2-way ANOVA with three independent variables - 'panel', 'replication' and 'sample'. If the descriptive attributes had significant differences among samples, Duncan's multiple range test was performed to identify the significance among samples at $p<0.05$. Principal component analysis (PCA) was conducted to determine the overall relationship between the descriptive attributes and the samples. Consumer acceptance test results were analyzed using 2-way ANOVA with two independent variables - 'panel' and 'sample'. If a significant difference was found among the samples, Duncan's multiple range test was conducted to determine the significance among samples at $p<0.05$. PCA for consumers' overall quality ratings were performed to identify consumer preference patterns toward samples. Partial least square (PLS) regression analysis was carried out by correlating the descriptive analysis and overall quality ratings to determine driving sensory attributes for frozen-cooked rice.

\section{RESULTS AND DISCUSSION}

\section{Quality characteristics of polished rice kernels}

Ratio of head rice, moisture content and freshness are presented in Table 2. BO and SI had less than $80 \%$ of head rice, which might negatively influence the consumer acceptability (Kim et al., 2000). Moisture contents of the rice kernels were below $16 \%$, which is the maximum allowable moisture content (NAQS, 2015). The degree of freshness of rice kernels were above 92 . The results showed that rice kernels in this study were fresh and lipid oxidation did not progress during the storage period (Matsukura et al., 2000).

\section{Descriptive analysis}

There were no significant difference in attributes for the 'Replication' variables ( $p$-value: $0.07-1.00)$. Panelists' responses were reproducible over the repeated evaluations and demonstrated the reliability of the panel training (Stone and Sidel, 2004). 'Sample' variables for each attribute were the major source of variations, with the exception of tap water (Results not shown). All attributes, except tap water, were significantly different among samples $(\phi<0.001)$. Since tap water did not differ significantly among the samples $(p=0.838)$, it was removed from further statistical analyses (Kwak et al. 2015a). Mean intensity ratings for 16 descriptive attributes are shown in Table 3 . In the aroma modality, IM had the strongest rice kernel aroma while BO had the strongest raw rice aroma. Sweetness was strongly perceived in $\mathrm{CH}$ and bitterness was strong in $\mathrm{BO}$. $\mathrm{BO}$ and $\mathrm{CH}$ had relatively high intensities for rice cake flavor. IL and SD comprised the lowest roasted flavor group in Duncan's multiple range test. In the texture modality,

$\begin{aligned} & \text { Table 2: Ratio of head rice kernels, moisture contents and } \\
& \text { degree of freshness of five polished rice kernels }\end{aligned}$
\begin{tabular}{lccc}
\hline Sample & Head rice (\%) & Moisture content (\%) & Freshness \\
\hline Boranchan (BO) & $75.73^{\mathrm{d}}$ & $14.50^{\mathrm{d}}$ & $93.3^{\mathrm{b}}$ \\
Chucheong (CH) & $95.37^{\mathrm{a}}$ & $15.77^{\mathrm{a}}$ & $92.0^{\mathrm{b}}$ \\
Shindongjin (SD) & $75.83^{\mathrm{d}}$ & $14.97^{\mathrm{c}}$ & $98.0^{\mathrm{a}}$ \\
IIpum (IL) & $83.10^{\mathrm{c}}$ & $15.17^{\mathrm{b}}$ & $93.7^{\mathrm{b}}$ \\
IImi (IM) & $88.10^{\mathrm{b}}$ & $15.67^{\mathrm{a}}$ & $98.7^{\mathrm{a}}$ \\
p-value & $<0.001$ & $<0.001$ & $<0.001$ \\
\hline
\end{tabular}

1)Different letters within a column meant significant differences at $p<0.05$ 
glutinous intensity was relatively low for IM and IL, while $\mathrm{BO}$ had the highest. Higher firmness was perceived for $\mathrm{BO}$ and $\mathrm{SD}$ while the perception of firmness for $\mathrm{CH}$ was lower. Chunkiness was strongly perceived in $\mathrm{CH}$ and $\mathrm{BO}$. IM had the highest particle feeling while IL had the lowest. $\mathrm{CH}$ had the highest perception of moistness, while $\mathrm{BO}, \mathrm{SD}$, IM had the lowest. Although the same milling ratio (92\%) was applied, BO had more embryo buds and could deliver a healthier image to consumers. The industry might use $\mathrm{BO}$ for developing a cooked rice product containing more embryo buds known as repository of nutrients (Butsat and Siriamornpun, 2010). SD had the highest intensities of glossiness, rice shape and size of rice. Whiteness was the lowest in $\mathrm{BO}$ and the same result was observed in asepticpackaged cooked rice (Kwak et al., 2015b).

In order to identify the overall relationship between the samples and their descriptive attributes, a PCA biplot was created and is shown in Figure 1. The biplot explained $48.42 \%$ for the first dimension (F1) and $27.51 \%$ for the second dimension (F2). The positive side of the first dimension was associated with firmness, amount of embryo buds, glutinousness, bitterness and raw rice aroma, while the negative side was related with sweetness, whiteness and moistness. The second dimension was characterized by glossiness, size of rice and rice shape on the positive side; while, rice kernel aroma, roasted flavor, rice cake flavor and particle feeling were loaded on the negative side. The PCA biplot presented a distinct separation of the five frozen-cooked rice samples. SD on the right-top quadrant

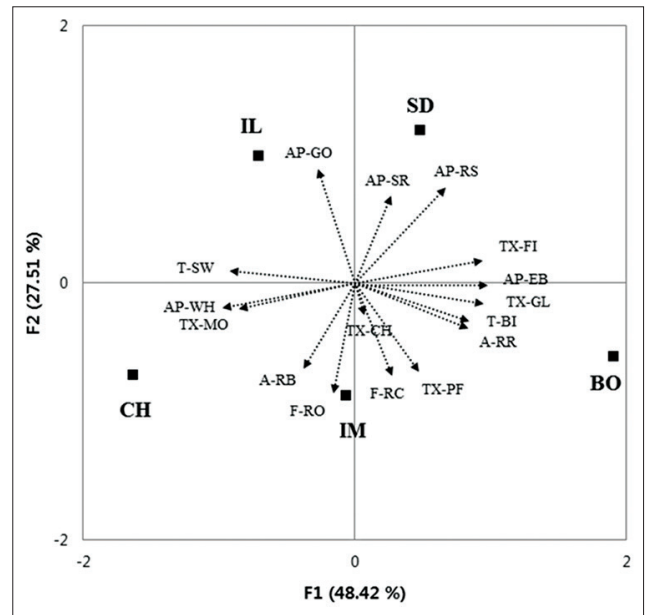

Fig 1. Principal component analysis 16 descriptive attributes for 5 frozen cooked rice samples. First letters A, F, T, TX, and AP from attributes mean aroma, flavor, taste, texture, and appearance, respectively. Arrows and squares represented descriptive attributes and samples, respectively. Sample codes referred to Table 2. Each abbreviation meant that $R B=$ rice bran, $R R=$ raw rice, $T A=\operatorname{tap}$ water, $S W=s w e e t$, $\mathrm{Bl}=$ bitter, $\mathrm{RC}=$ rice cake, $\mathrm{RO}=$ roasted, $\mathrm{GL}=$ glutinousness, $\mathrm{Fl}=$ firmness, $\mathrm{CH}=$ chunkiness, $\mathrm{PF}=$ particle feeling, $\mathrm{MO}=$ moistness, $\mathrm{EB}=$ amount of embryo buds, $\mathrm{GO}=$ glossiness, $\mathrm{RS}=$ rice shape, $\mathrm{WH}=$ whiteness, and $\mathrm{SR}=$ size of rice. was related with size of rice and rice shape. $\mathrm{BO}$ was located on the right side of the biplot and associated with raw rice aroma, bitterness and glutinousness. IM was at the bottom of the biplot and characterized by roasted and rice cake flavors. $\mathrm{CH}$ was loaded on the left-bottom quadrant and showed positive correlations with moistness, whiteness and sweetness. IL was presented on the top-left quadrant of the biplot and associated with glossiness. Throughout the descriptive analysis rice varieties had different sensory profiles, which might influence to consumer acceptability.

\section{Consumer acceptance test}

Consumer acceptance ratings for overall quality, appearance, aroma, texture, levels of glutinousness and hardness are presented in Table 4. IL showed the highest mean overall quality rating (11.08) and had a statistical significance from the other samples $\left(\mathrm{F}_{4,205}=4.519, p=0.003\right)$. IM was the

Table 3: Intensity of $\mathbf{1 6}$ descriptive sensory attributes for frozen-cooked rice samples

\begin{tabular}{|c|c|c|c|c|c|c|}
\hline \multirow[t]{2}{*}{ Attribute } & \multicolumn{5}{|c|}{ Sample } & \multirow[t]{2}{*}{$p$-value } \\
\hline & BO') & SD & IM & IL & $\mathrm{CH}$ & \\
\hline \multicolumn{7}{|l|}{ Aroma } \\
\hline Rice bran & $1.03^{\mathrm{c} 2)}$ & $1.13^{c}$ & $2.69^{a}$ & $1.03^{c}$ & $1.88^{b}$ & $<0.001$ \\
\hline Raw rice & $4.56^{a}$ & $1.19^{b c}$ & $1.50^{\mathrm{b}}$ & $1.19^{b c}$ & $1.06^{c}$ & $<0.001$ \\
\hline \multicolumn{7}{|l|}{ Taste } \\
\hline Sweet & $3.06^{c}$ & $5.19^{b}$ & $4.63^{b}$ & $5.06^{b}$ & $6.75^{a}$ & $<0.001$ \\
\hline Bitter & $4.50^{\mathrm{a}}$ & $1.75^{b}$ & $1.63^{b}$ & $1.50^{\mathrm{b}}$ & $1.50^{b}$ & $<0.001$ \\
\hline \multicolumn{7}{|l|}{ Flavor } \\
\hline Rice cake & $8.75^{a}$ & $5.25^{d}$ & $5.81^{\circ}$ & $4.25^{e}$ & $8.06^{b}$ & $<0.001$ \\
\hline roasted & $5.69^{b}$ & $3.06^{d}$ & $4.81^{\circ}$ & $2.88^{d}$ & $7.06^{a}$ & $<0.001$ \\
\hline \multicolumn{7}{|l|}{ Texture } \\
\hline Glutinousness & $10.63^{a}$ & $8.94^{b}$ & $8.63^{b}$ & $5.88^{c}$ & $5.69^{c}$ & $<0.001$ \\
\hline Firmness & $8.44^{\mathrm{a}}$ & $8.19^{a}$ & $7.00^{b}$ & $5.94^{c}$ & $4.88^{d}$ & $<0.001$ \\
\hline Chunkiness & $8.63^{a}$ & $6.88^{b}$ & $4.81^{d}$ & $5.81^{c}$ & $9.06^{a}$ & $<0.001$ \\
\hline Particle feeling & $6.44^{b}$ & $5.19^{c}$ & $7.56^{a}$ & $3.88^{d}$ & $4.94^{c}$ & $<0.001$ \\
\hline Moistness & $5.69^{\circ}$ & $5.69^{c}$ & $5.94^{\circ}$ & $7.19^{b}$ & $9.13^{a}$ & $<0.001$ \\
\hline \multicolumn{7}{|l|}{ Appearance } \\
\hline Amount of embryo buds & $8.50^{\mathrm{a}}$ & $6.56^{b}$ & $5.06^{c}$ & $2.81^{d}$ & $1.94^{\mathrm{e}}$ & $<0.001$ \\
\hline Glossiness & $4.44^{d}$ & $8.31^{a}$ & $3.69^{\mathrm{e}}$ & $7.19^{b}$ & $6.13^{c}$ & $<0.001$ \\
\hline Rice shape & $8.38^{b}$ & $9.94^{\mathrm{a}}$ & $5.75^{c}$ & $8.00^{\mathrm{b}}$ & $4.13^{d}$ & $<0.001$ \\
\hline Whiteness & $5.81^{d}$ & $7.50^{c}$ & $8.81^{b}$ & $8.69^{b}$ & $11.00^{\mathrm{a}}$ & $<0.001$ \\
\hline Size of rice & $8.00^{b}$ & $9.69^{a}$ & $4.25^{d}$ & $7.44^{\mathrm{bc}}$ & $7.19^{c}$ & $<0.001$ \\
\hline
\end{tabular}

${ }^{1)}$ Sample codes referred to Table 2. ${ }^{2}$ Different letters within a row meant significant differences at $p<0.05$

Table 4: Mean consumer acceptance ratings of frozen-cooked rice samples ${ }^{1)}$

\begin{tabular}{lcccccc}
\hline Acceptance & BO $^{2)}$ & SD & IM & IL & CH & $p$-value \\
\hline Overall & $9.90^{\mathrm{b} 3)}$ & $9.60^{\mathrm{b}}$ & $9.42^{\mathrm{b}}$ & $11.08^{\mathrm{a}}$ & $10.04^{\mathrm{b}}$ & 0.003 \\
Appearance & $10.37^{\mathrm{b}}$ & $10.02^{\mathrm{b}}$ & $9.62^{\mathrm{b}}$ & $11.13^{\mathrm{a}}$ & $10.38^{\mathrm{b}}$ & 0.002 \\
Aroma & $10.37^{\mathrm{a}}$ & $10.04^{\mathrm{a}}$ & $9.94^{\mathrm{a}}$ & $10.56^{\mathrm{a}}$ & $10.35^{\mathrm{a}}$ & 0.368 \\
Texture & $9.67^{\mathrm{b}}$ & $9.75^{\mathrm{b}}$ & $9.04^{\mathrm{b}}$ & $11.04^{\mathrm{a}}$ & $9.75^{\mathrm{b}}$ & 0.000 \\
Glutinousness & $9.50^{\mathrm{bc}}$ & $9.08^{\mathrm{bc}}$ & $9.00^{\mathrm{c}}$ & $10.60^{\mathrm{a}}$ & $9.77^{\mathrm{b}}$ & 0.003 \\
Level of cooking & $9.42^{\mathrm{b}}$ & $9.48^{\mathrm{b}}$ & $9.25^{\mathrm{b}}$ & $10.56^{\mathrm{a}}$ & $9.25^{\mathrm{b}}$ & 0.041 \\
\hline
\end{tabular}

${ }^{1)}$ Mean separation test was conducted using Duncan's multiple range test at $p<0.05 .{ }^{2)}$ Sample codes referred to Table 2. ${ }^{3)}$ Same letters within a row mean there was no statistical difference at $p<0.05$ 
least accepted sample with a mean average rating of 9.42 . Appearance $\left(\mathrm{F}_{4,205}=4.096, p=0.002\right)$, texture $\left(\mathrm{F}_{4,205}=5.468\right.$, $p<0.001)$, levels of glutinousness $\left(\mathrm{F}_{4,205}=4.102, p=0.003\right)$ and levels of cooking $\left(\mathrm{F}_{4,205}=2.541, p=0.041\right) \mathrm{had}$ statistical significance. Only aroma acceptance showed no significant difference among the samples $\left(\mathrm{F}_{4,205}=1.079\right.$, $p=0.368)$, meaning that different rice varieties did not result in differences in aroma acceptance for frozen-cooked rice. IL received the highest acceptance ratings for the other attributes. The second most accepted sample based on the acceptance ratings was $\mathrm{CH}$. IM had the lowest acceptance attributes.

PCA was conducted to identify the direction of consumer preference towards frozen-cooked rice samples. The PCA biplot of overall quality rating results explained 62.15\% of the total variation (Figure 2). The first dimension (F1) explained $36.28 \%$ and the second dimension (F2) explained $25.86 \%$ of the total variation. The majority of consumers were placed near the right and right-top areas of the biplot where IL and $\mathrm{CH}$ were located. Fewer consumers were placed towards the left-top areas where IM and SD were located. The consumer preference pattern was identical to the mean consumer acceptance rating pattern. One interesting point from the mean consumer acceptance ratings and the biplot was that SD and IM have been used for frozen-cooked rice processing (Kwak et al., 2013a), but received lower consumer acceptance. On the other hand, $\mathrm{CH}$ and IL, which are consumed as steamed rice at home, had the highest consumer acceptance. Glossiness, sweetness, whiteness and moistness, which showed positive correlations with IL and $\mathrm{CH}$ in Figure 1, might affect consumer preference positively.

\section{Driving sensory attributes}

To determine the driving sensory attributes for overall quality ratings by consumers, PLS regression analysis was conducted using the descriptive analysis and overall quality ratings (Tenenhaus et al., 2005). A correlation map is presented in Figure 3 showing the relationships among overall quality, descriptive attributes and samples. The $x$-axis ( $\mathrm{t} 1$ ) explained $49.57 \%$ and the $y$-axis ( $\mathrm{t} 2$ ) explained $24.32 \%$ of the total variations. The overall quality rating was positively correlated with moistness and negatively correlated with firmness, amount of embryo buds, glutinousness and particle feeling. Highly accepted samples (IL and $\mathrm{CH}$ ) were close to overall quality on the correlation map, whereas less accepted samples (BO, IM and SD) were located opposite side from the overall quality.

Variable importance in the projection (VIP) values by the PLS regression analysis represented significant variables for the model (Indahl et al., 2009). Generally, VIP values greater than 1 considered influential factors in PLS regression

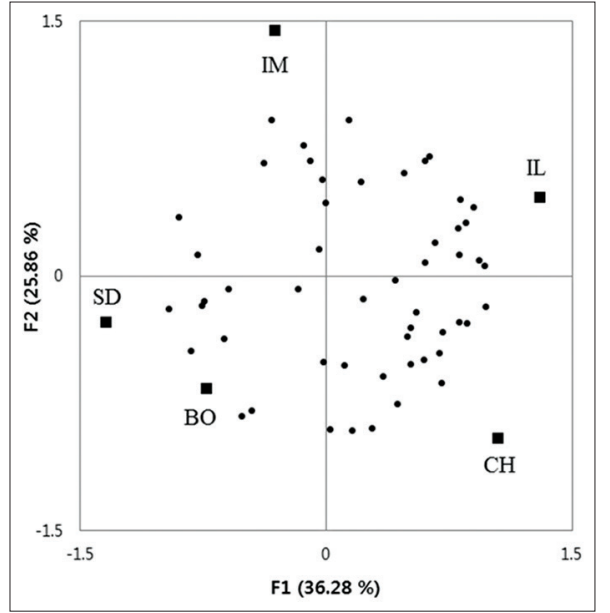

Fig 2. Principal component analysis of 5 frozen cooked rice samples for overall quality ratings by 52 consumers. Squares and circles represented samples and consumers, respectively. Sample codes referred to Table 2 .

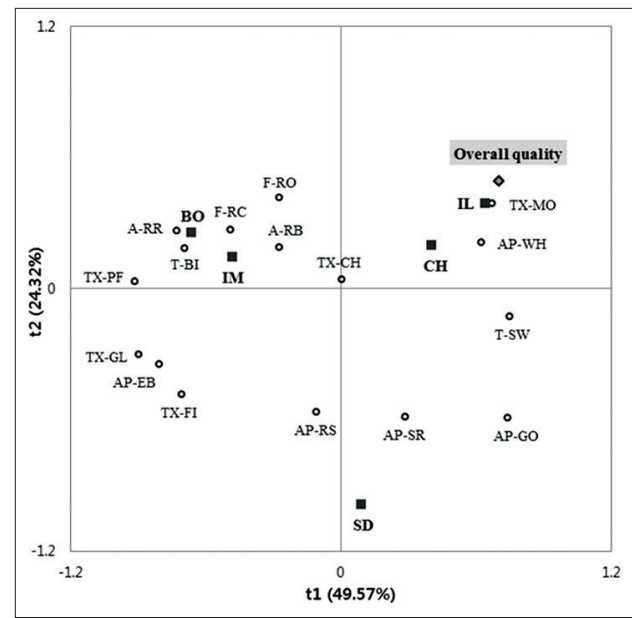

Fig 3. Correlation map for mean overall quality ratings from 52 consumers and 16 descriptive sensory attributes of 5 frozen-cooked rice samples. First letters A, F, TA, T and AP mean aroma, flavor, taste, texture, and appearance, respectively. A diamond, circles and squares meant overall quality rating, descriptive attributes and samples. First letters A, F, T, TX, and AP from attributes mean aroma, flavor, taste, texture, and appearance, respectively. A dot means a descriptive attribute. A square means a cooked rice sample. Each abbreviation means that $\mathrm{RB}=$ rice bran, $\mathrm{RR}=$ raw rice, $\mathrm{TA}=$ tap water, $\mathrm{SW}=$ sweet, $\mathrm{Bl}=$ bitter, $\mathrm{RC}=$ rice cake, $\mathrm{RO}=$ roasted, $\mathrm{GL}=$ glutinousness, $\mathrm{Fl}=$ firmness, $\mathrm{CH}=$ chunkiness, $\mathrm{PF}=$ particle feeling, $\mathrm{MO}=$ moistness, $\mathrm{EB}=$ amount of embryo buds, $\mathrm{GO}=$ glossiness, $\mathrm{RS}=$ rice shape, $\mathrm{WH}=$ whiteness, and $\mathrm{SR}=$ size of rice.

models (Wold, 2008; Azizan et al., 2012). In this study, the authors determined the driving sensory attributes as VIP values of 1 or above in $\mathrm{t} 1$ and $\mathrm{t} 2$ (Table 5) as well as the position in the correlation map (Figure 3). Rice bran aroma, glutinousness, firmness, particle feeling and amount of embryo bud were the drivers of disliking attributes for the frozen-cooked rice. Consumers seemed to be more influenced by negative factors than by positive factors in their determination of the overall quality of frozen-cooked 


\begin{tabular}{|c|c|c|c|}
\hline \multirow[t]{2}{*}{ Attribute } & \multicolumn{2}{|c|}{$\begin{array}{l}\text { Variable importance in } \\
\text { the projection }\end{array}$} & \multirow[t]{2}{*}{$\begin{array}{l}\text { Driving } \\
\text { attribute }\end{array}$} \\
\hline & $\begin{array}{c}\text { t1 (horizontal } \\
\text { effect) }\end{array}$ & $\begin{array}{c}\text { t2 (vertical } \\
\text { effect) }\end{array}$ & \\
\hline \multicolumn{4}{|l|}{ Aroma } \\
\hline Rice bran & 1.352 & 1.445 & Negative \\
\hline Raw rice & 0.366 & 1.069 & \\
\hline \multicolumn{4}{|l|}{ Taste } \\
\hline Sweet & 0.354 & 1.128 & \\
\hline Bitter & 0.384 & 0.995 & \\
\hline \multicolumn{4}{|l|}{ Flavor } \\
\hline Rice cake & 0.881 & 0.722 & \\
\hline Roasted & 0.774 & 0.703 & \\
\hline \multicolumn{4}{|l|}{ Texture } \\
\hline Glutinousness & 1.588 & 1.301 & Negative \\
\hline Firmness & 1.222 & 1.003 & Negative \\
\hline Cohesiveness of mass & 0.027 & 0.047 & \\
\hline Particle feeling & 2.056 & 1.740 & Negative \\
\hline Moistness & 1.057 & 0.884 & \\
\hline \multicolumn{4}{|l|}{ Appearance } \\
\hline Amount of embryo buds & 1.296 & 1.077 & Negative \\
\hline Glossiness & 0.974 & 0.894 & \\
\hline Rice shape & 0.159 & 0.405 & \\
\hline Whiteness & 0.418 & 0.847 & \\
\hline Size of rice & 0.449 & 0.376 & \\
\hline
\end{tabular}

rice, as there was no drivers of liking attribute. Moistness appeared to have a strong positive relation with overall quality; however, its VIP value of $\mathrm{t} 2$ did not meet the requirements to be a driver of liking. Existence of only drivers of disliking for frozen-cooked rice would be acceptable since rice is generally consumed right after cooking in Korea. Freezing and re-heating cooked rice might result in a negative image for the quality of the cooked rice (Cho and Koh, 2003). Since the consumers knew the testing item is frozen-cooked rice, it can be expected that they would have negative impressions for the samples.

The strongest disliking factor was particle feeling perceived while eating the frozen-cooked rice samples and this factor had the highest VIP value (Table 5). Particle feeling might be associated with the perception of half-cooked rice; therefore, consumers might feel that the samples were not fully cooked. In addition, the re-heating process (three-minute microwave for $200 \mathrm{~g}$ of frozen-cooked rice) dried the surface of the samples. Consumers might perceive the particle feeling when chewing the dried surface of the frozen-cooked rice. Another interesting point for the frozen-cooked rice was glutinousness, which was considered a factor for disliking. The opposite result was found in aseptic-packaged cooked rice (Kwak et al., 2015) as well as in a cooked rice processing study (Kwak et al., 2013a), with glutinousness categorized as a driver of liking. The glutinousness perceived in the frozen-cooked rice might come from different physical properties in comparison with aseptic-packaged cooked rice or steamed rice. The re-heating process in a microwave dries the surface of frozen-cooked rice while the center remains moist (Zhao et al., 2007). The different texture conditions between the surface and the center of frozen-cooked rice kernel would influence the perception of glutinousness in frozen-cooked rice. Consumers seemed to not prefer this type of glutinousness and considered it as a disliking factor.

\section{CONCLUSIONS}

Rice varieties significantly influenced to sensory profiles and consumer acceptability of frozen-cooked rice samples. Therefore, careful selection of rice varieties is necessary in order to produce high quality frozen-cooked rice. IL was the most preferred in the consumer acceptance test. This variety would be the best choice among the five major rice varieties tested for frozen-cooked rice processing. The industry should consider IL as the first option for frozen-cooked rice. The driving sensory attributes for frozen-cooked rice were negatively correlated with the overall quality ratings in PLS regression analysis. This was the supporting evidence that consumer acceptability was heavily dependent on the disliking characteristics of the samples. Frozen-cooked rice producers should focus on eliminating of the drivers of disliking attributes (rice bran aroma, glutinousness, firmness, particle feeling, and amount of embryo buds) to ensure consumer satisfaction for their frozen-cooked rice products.

\section{ACKNOWLEDGEMENT}

The present research was conducted by the research fund of Dankook University in 2014.

\section{Authors' contributions}

Study concept and management: H. S. K., M. K. Conducted the experiments: H. S. K., Y. J., M. K. Analysis and interpretation of data: H. S. K., Y. J., M. K. Drafting of manuscript: H. S. K., Y. J. Critical revision: M. K.

\section{REFERENCES}

AOAC. 2002. Official Methods of Analysis, 16th ed. Association of Official Analytical Chemists, Washington, DC.

Azizan, K. A., S. N. Baharum, H. W. Ressom and N. M. Noor. 2012. GC-MS analysis and PLS-DA validation of the trimethyl silylderivatization techniques. Am. J. Appl. Sci. 9: 1124-1136.

Bárcenas, M. E. and C. M. Rosell. 2006. Effect of frozen storage time on the bread crumb and aging of par-baked bread. Food Chem. 95: 438-445. 
Butsat, S. and S. Siriamornpun. 2010. Antioxidant capacities and phenolic compounds of the husk, bran and endosperm of Thai rice. Food Chem. 119: 606-613.

Chen, T. F. and C. L. Chen. 2003. Analyzing the freshness of intact rice grains by colour determination of peroxidase activity. J. Sci. Food Agri. 83: 1214-1218.

Cho, J. H. and B. K. Koh. 2003. A survey on the rice-based processed food consumption of the housewives at Daeju. Korean J. Soc. Food Cookery Sci. 19: 300-307.

De Kock, S., A. Minnaar, D. Berry and J. R. N. Taylor. 1995. The effect of freezing rate on the quality of cellular and non-cellular parcooked starchy convenience foods. LWT-Food Sci. Technol. 28: 87-95.

Food News. 2014. Rice variety development for processed cooked rice. Available from: http://www.foodnews.co.kr/news/ articleView.html?idxno=50402. [Last accessed on 2015 Feb 10].

Indahl, U. G., K. H. Liland and T. Næs. 2009. Canonical partial least squares: A unified PLS approach to classification and regression problems. J. Chemometr. 23: 495-504.

Ji, Y. 2010. Increasing frozen-cooked rice market with big food companies. Agric. Fish. Livest. News. 21: 7.

Kim, S. S., S. E. Lee, O. W. Kim and D. C. Kim. 2000. Physicochemical characteristics of chalky kernels and their effects on sensory quality of cooked rice. Cereal Chem. 77: 376-379.

Ki Yong, H., K. Jae Kwon, N. Jeong Kwon, K. Jong Cheol, K. Ki Yeong, K. Hyeon Jung, S. Mun Sik, B. Man Kee, K. Bo Kyeong, K. Woo Jae, S. Woon Coul, P. Hyun Su. 2012. A new medium-late japonica rice cultivar, "'Boramchanwith' super high yield. Korean J. Breed. Sci. 44: 346-350.

Kum, J. S. 2010. Increasing of rice consumption by innovating grain processing technology. Food Preserv. Process. Ind. 9: 49-59.

Kwak, H. S., H. G. Kim, H. S. Kim, Y. S. Ahn, K. Jung, H. Y. Jeong and T. H. Kim. 2013a. Sensory characteristics and consumer acceptance of frozen cooked rice by rapid freezing process compared to home-made and aseptic packaged cooked rice. Prev. Nutr. Food Sci. 18: 67-75.

Kwak, H. S., Y. J. Oh, H. B. Kang and T. H. Kim. 2013b. Descriptive profile and liking/disliking factors for aseptic-packaged rice porridge. J. Korean Soc. Food Sci. Nutr. 42: 1878-1885.

Kwak, H. S., B. H. Ahn, H. R. Kim and S. Y. Lee. 2015a. Identification of senory attributes that drive the likeability of Korean rice wines by American panelists. J. Food Sci. 80: S161-S170.

Kwak, H. S., M. Kim, Y. Lee and Y. Jeong. 2015b. Identification of key sensory attributes for consumer acceptance and instrumental quality of aseptic-packaged cooked rice. Int. J. Food Sci. Technol. 50: 691-699.

Matsukura, U., S. Kaneko and M. Momma. 2000. Method for measuring the freshness of individual rice grains by means of a color reaction of catalase activity. Jpn. Soc. Food Sci. Technol. 47: 523-528.

Mazzucchelli, R. and J. Guinard. 1999. Comparison of monadic and simultaneous sample presentation methods in a descriptive analysis of milk chocolate. J. Sens. Stud. 14: 235-248.

Meullenet, J. F., V. K. Griffin, K. Carson, G. Davis, S. Davis, J. A. Gross, E. Hankins, E. Sailer, C. Sitakalin, S. Suwansri and A. V. Caicedo. 2001. Rice external preference mapping for Asian consumers living in the United States. J. Sens. Stud. 16: 73-94.

National Agricultural Product Management Quality Service, NQAS. 2014. Quality Standard of Rice. Available from: http://www.naqs. go.kr/information/info_08.jsp. [Last accessed on 2015 Jan 19].

Oh, S. K., D. J. Kim, A. R. Cheun, M. R. Yoon, H. C. Hong, I. M. Choi, Y. J. Oh, K. B. Oh and Y. K. Kim. 2010. Quality evaluation of Juanbyeo as aseptic-packaged cooked rice. Korean J. Food Sci. Technol. 42: 721-726.

Olivera, D. F. and V. O. Salvadori. 2009. Effect of freezing rate in textural and rheological characteristics of frozen cooked organic pasta. J. Food Eng. 90: 271-276.

Park, S. W. 2008. Rice for processing food industry. Food J. Seoul. 19: $28-41$.

Stone, H. and J. L. Sidel. 2004. Sensory Evaluation Practices, Elsevier Academic Press, San Diego, Pp. 162-275.

Suwansri, S., J. F. Meullenet, J. A. Hankins and K. Griffin. 2002. Preference mapping of domestic/imported jasmine rice for US-Asian Consumers. J. Food Sci. 67: 2420-2431.

Tenenhaus, M., J. Pagès, L. Ambroisine and C. Guinot. 2005. PLS methodology to study relationships between hedonic judgements and product characteristics. Food Qual. Prefer. 16: 315-325.

Wakeling, I. N. and H. J. MacFie. 1995. Designing consumer trials balanced for first and higher orders of carry-over effect when only a subset of k samples from t may be tested. Food Qual. Prefer. 6: 299-308.

Williams, E. G. 1949. Experimental designs balanced for the estimation of residual effects of treatments. Aust. J. Chem. 2: 149-158.

Wold, S., L. Eriksson and S. Clementi. 2008. Statistical validation of QSAR results. In: Waterbeemd, H (Ed.), Chemometric Methods in Molecular Design, John Wiley \& Sons, Hoboken, Pp. 309-338.

Yau, N. J. N. and J. J Huang. 1996. Sensory analysis of cooked rice. Food Qual. Prefer. 7: 263-270.

Yu, S., Y. Ma and D. W. Sun. 2010. Effects of freezing rates on starch retrogradation and textural properties of cooked rice during storage. LWT-Food Sci. Technol. 43: 1138-1143.

Zhao, S., S. Xiong, C. Qiu and Y. Xu. 2007. Effect of microwaves on rice quality. J. Stored Prod. Res. 43: 496-502. 